\title{
Searching for AGN among unidentified INTEGRAL sources
}

\section{E. Maiorano ${ }^{* a}$, R. Landi ${ }^{a}$, J.B. Stephen ${ }^{a}$, L. Bassani ${ }^{a}$, N. Masetti ${ }^{a}$, P. Parisi ${ }^{a}$, P.} Palazzi $^{a}$, P. Parma ${ }^{b}$, A.J. Bird $^{c}$, A. Bazzano ${ }^{d}$, P. Ubertini ${ }^{d}$, E. Jiménez-Bailón ${ }^{e}$, V. Chavushyan $^{f}$, G. Galaz ${ }^{g}$, D. Minniti ${ }^{g, h}$, L.Morelli $^{i}$

${ }^{a}$ INAF-IASF Bologna,

Via Gobetti 101, 40129 Bologna (Italy)

${ }^{b}$ INAF-IRA Bologna,

Via Gobetti 101, 40129 Bologna (Italy)

${ }^{c}$ Univ. Southampton,

School of Physics and Astronomy, University of Southampton, SO17 1BJ(UK)

${ }^{d}$ INAF-IASF Roma,

Via Fosso del Cavaliere 100, 00133 Rome (Italy)

${ }^{e}$ UNAM Mexico City,

México, Apartado Postal 70-264, 04510 México D.F. (México)

${ }^{f}$ INAOE Puebla,

Apartado Postal 51-216, 72000 Puebla (México)

${ }^{g}$ PUC Santiago de Chile,

Universidad Católica de Chile, Casilla 306, Santiago 22 (Chile)

${ }^{h}$ Specola Vaticana,

Specola Vaticana, V-00120 (Città del Vaticano)

${ }^{i}$ Univ. Padova (Italy),

Vicolo dell'Osservatorio 3, I-35122 Padua, Italy

E-mail: maioranodiasfbo.inaf.it

\begin{abstract}
We report on a new method to identify active galactic nuclei (AGN) among unidentified INTEGRAL sources. This method consists of cross-correlating unidentified objects listed in the fourth IBIS survey with infrared (IR) and radio catalogues to look for possible counterparts and then verifying, by means of X-ray and optical follow-up observations, the likelihood of these associations. In particular we discuss here a sample of eight objects, six of which have been possibly identified as AGN.
\end{abstract}

The Extreme and Variable High Energy Sky

September 19-23, 2011

Chia Laguna (Cagliari), Italy

${ }^{*}$ Speaker. 


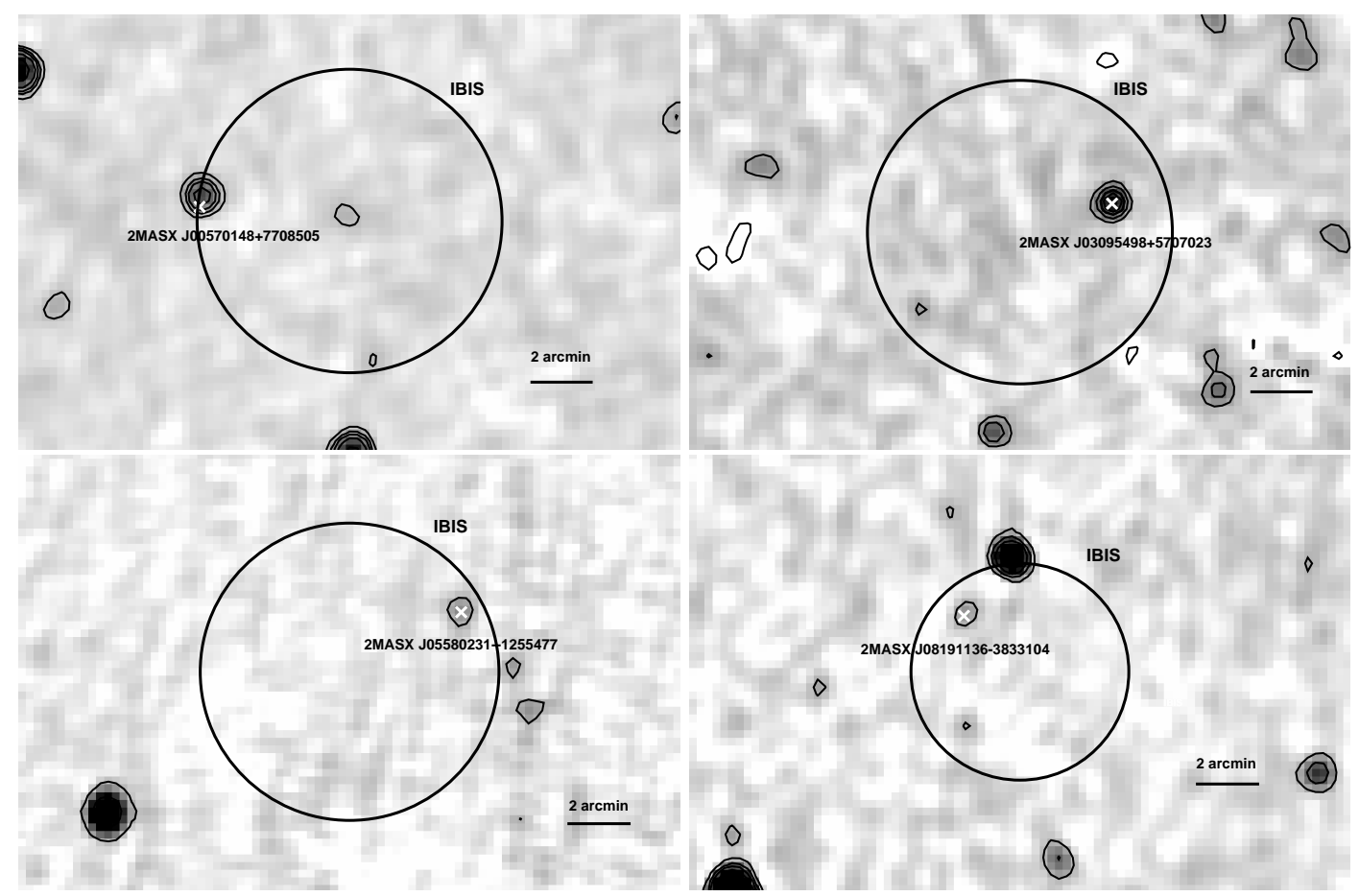

Figure 1: NVSS image cut-outs for four sources in our sample with overimposed IBIS error circle and 2MASX source positions. In all images, the north is up and east to the left. The scale is reported at the bottom right corner.

\section{Introduction}

Historically, AGN were discovered with radio observations, i.e. the radio selection is often a way to recognize active galaxies, except at very low luminosities where star formation in galaxies can also produce radio emission; radio data can therefore be used to recognize AGN among unidentified INTEGRAL sources. Contamination from starburst galaxies is not an issue, since no pure starburst galaxy has so far been detected by INTEGRAL. Cross-correlation with galaxy catalogues available for example in the IR band allows to select those objects which are associated to galaxies thus avoiding contamination from galactic sources with radio emission. Following this reasoning we have cross-correlated our set of unidentified INTEGRAL sources in the fourth IBIS catalogue [1] with radio/IR catalogues in order to extract a sample of objects likely associated with an AGN.

\section{Sample Selection}

For the IR bands we have used the 2MASX Source Catalogue [2] which is a powerful tool to identify sources associated with galaxies. Indeed $97 \%$ of the objects contained in the 2MASX survey are galaxies, while the remaining $3 \%$ is made of galactic objects not expected to emit in the IBIS band. As radio catalogues we have used the National Radio Astronomy Observatories Very Large Array Sky Survey (NVSS; [阬) and the Sydney University Molonglo Sky Survey (SUMSS; [凹): they are similar in sensitivity and spatial resolution, and together they cover the whole sky. 


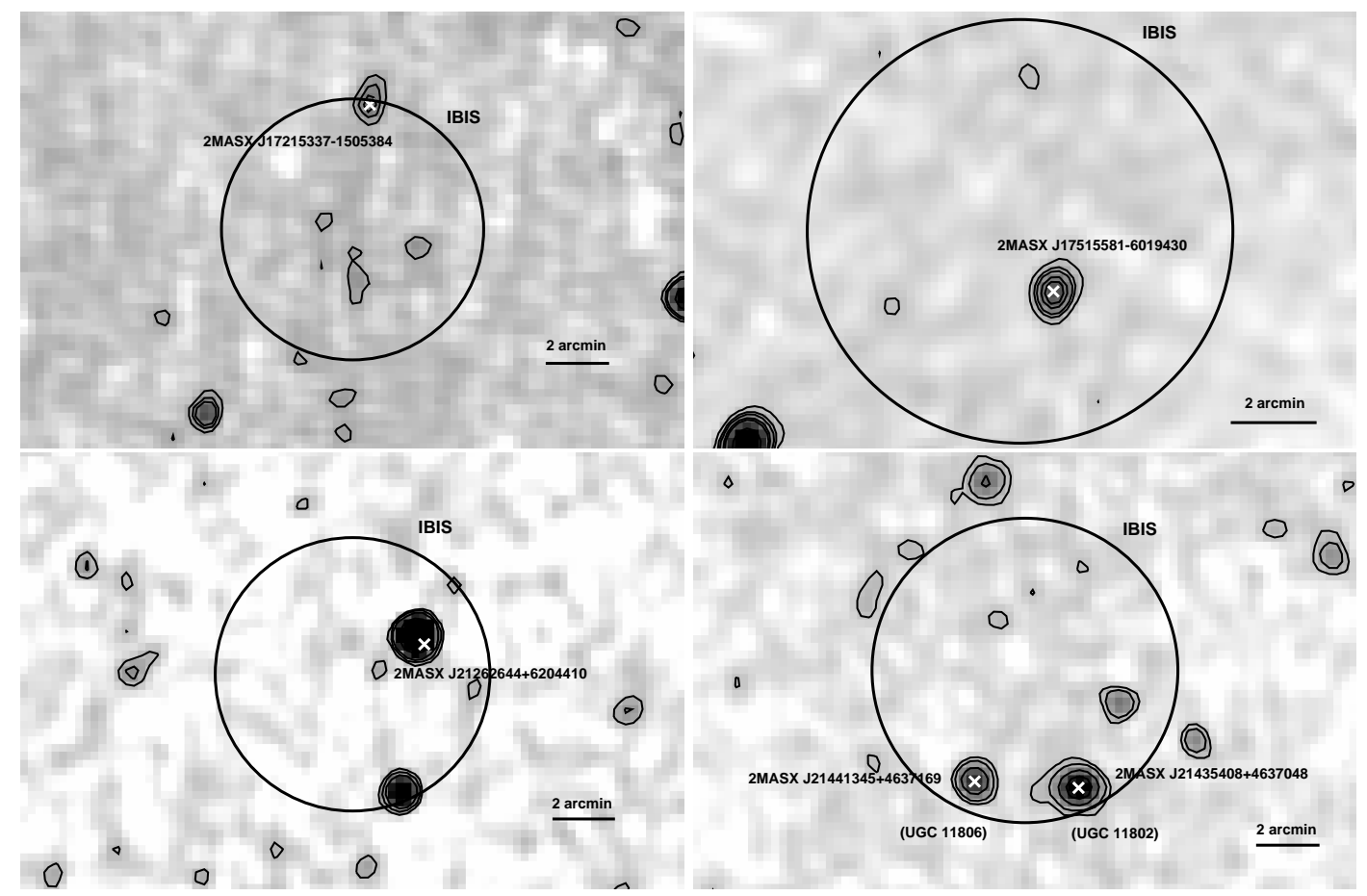

Figure 2: NVSS or SUMSS image cut-outs for four sources in our sample with overimposed IBIS error circle and 2MASX source positions. In all images, the north is up and east to the left. The scale is reported at the bottom right corner.

Table 1: IBIS, 2MASX and radio positions (and corresponding errors) for each source in the sample. The radio positions come from the analysis carried out in Maiorano et al. (2011) where the IBIS error box radius for each source is also reported.

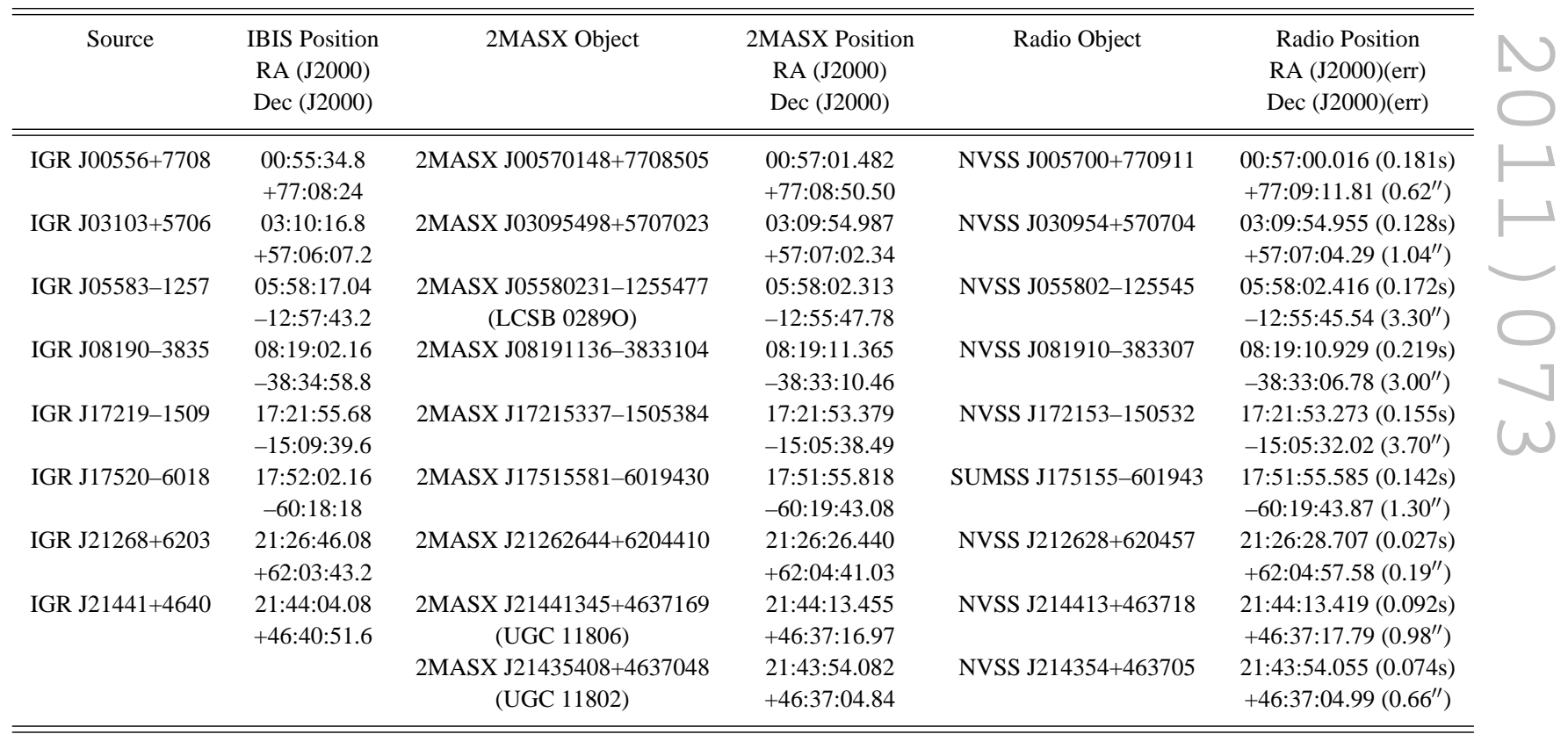


The radio detection provides evidence that a 2MASX galaxy is likely an AGN and so the possible counterpart of the IBIS source. To perform the correlation, we used the standard statistical method employed very successfully in other cases ([5], [6], [7]) and which is extensively described for this case in Maiorano et al. (2011). By means of a sequence of cross-correlations we extracted a final sample of eight objects which are seen in all three wavebands (hard X-rays, IR and radio); all eight can be considered as AGN candidates because they are classified as galaxies in the 2MASX catalogue and are detected both in radio and hard X-rays. All objects of the sample are reported in Table 1: for each source we list theINTEGRAL name, the IBIS position, the IR (2MASX) and radio (NVSS or SUMSS) positions and associated errors of the putative counterparts.

Figs 1 and 2 show the collection of NVSS/SUMSS image cut-outs for all of our sources with overimposed IBIS error circle and 2MASX source position. In order to confirm the proposed counterparts we have made a set of X-ray and optical follow up observations: the former were made with the XRT telescope on board Swift, while the latter were performed at 1.5-m CTIO telescope (Chile) and 2.1-m San Pedro Martir telescope (Mexico).

\section{Results}

In the following we describe in some details three of the eight objects of the sample, while information on the entire sample can be found in Maiorano et al. (2011).

\subsection{IGR J08190-3835}

Within the IBIS uncertainty, XRT detects only one source with a statistical significance of $5.8 \sigma$ in the energy range $0.3-10 \mathrm{keV}$ and $5.6 \sigma$ above $3 \mathrm{keV}$. This object is positionally coincident with both 2MASX and NVSS sources (Fig. 1, bottom-right panel). The radio analysis provides a flux of $\sim 3 \mathrm{mJy}$ at $20 \mathrm{~cm}$. The X-ray data are well described by an absorbed power law with a photon index fixed to 1.8 and an obseved 2-10 keV flux of $1.5 \times 10^{-12} \mathrm{erg} \mathrm{cm}^{-2} \mathrm{~s}^{-1}$; the intrinsic column density is $\mathrm{N}_{\mathrm{H}}($ intr $) \sim 1.4 \times 10^{23} \mathrm{~cm}^{-2}$, which exceedes the Galactic value of $9.6 \times 10^{21}$ $\mathrm{cm}^{-2}$ [9]. The optical spectrum (Fig. 4, left panel) shows $\mathrm{H}_{\alpha}$, [N II] and [S II] narrow emission lines at redshift $z=0.009 \pm 0.001$ superimposed on a very reddened continuum. The flux ratios among these emission features suggest that the object is a Type 2 AGN. Overall, we conclude that the proposed 2MASX/NVSS/XRT source is the actual counterpart of the IBIS detected object and it is a narrow-line (obscured) AGN.

\subsection{IGR J1752-1860}

An X-ray source is well detected at $13.6 \sigma$ confidence level in the range $0.3-10 \mathrm{keV}$ by XRT within the IBIS error box. It coincides with both the 2MASS extended object and the radio source reported in the SUMSS (Fig. 2, top-right panel). The source is detected in radio at $36 \mathrm{~cm}$ with a flux of $25.2 \mathrm{mJy}$. The X-ray data are well reproduced by an absorbed power law with fixed photon index of 1.8 and an observed 2-10 keV flux of $2.6 \times 10^{-12} \mathrm{erg} \mathrm{cm}^{-2} \mathrm{~s}^{-1}$; the intrinsic column density is $\mathrm{N}_{\mathrm{H}}($ intr $)=1.3 \times 10^{23} \mathrm{~cm}^{-2}$ which exceedes the Galactic value of $7 \times 10^{20} \mathrm{~cm}^{-2}$. This source is also reported in the BAT 58-Month catalogue ${ }^{1}$ as SWIFT J1751.8-6019, with flux $1.7 \times 10^{-11}$ erg cm${ }^{-2} \mathrm{~s}^{-1}$ in the range $14-195 \mathrm{keV}$. Based on all the above information, we conclude that this

\footnotetext{
${ }^{1}$ http://swift.gsfc.nasa.gov/docs/swift/results/bs58mon/
} 

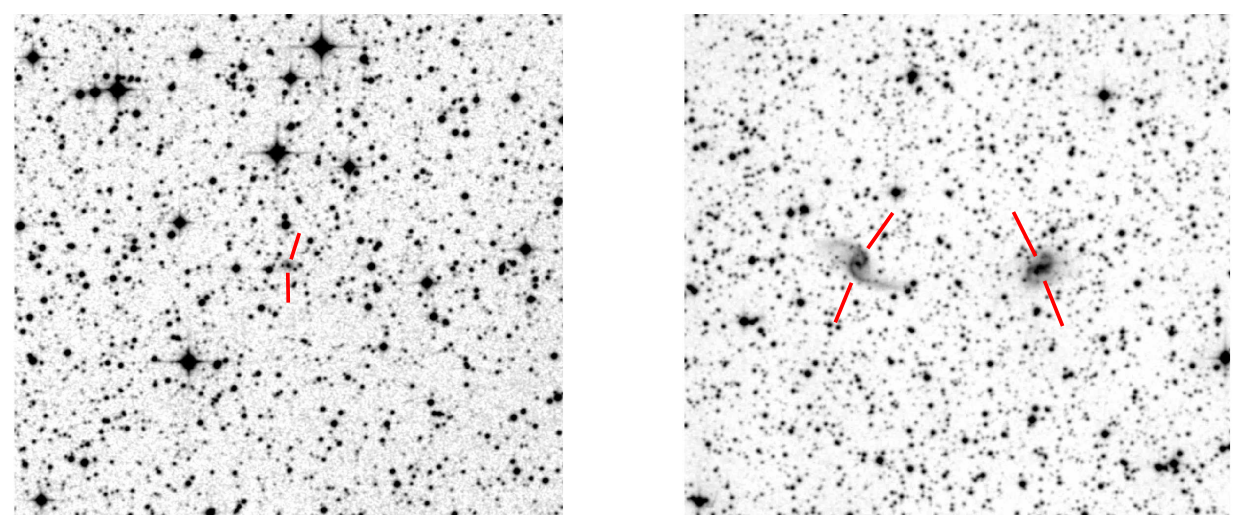

Figure 3: Optical images of the fields of 2 of the INTEGRAL hard X-ray sources selected in this work for optical spectroscopic follow-up: IGR J08190-3835 (left panel) and IGR J21441+4640 (right panel). The proposed optical counterparts are indicated with tick marks. Field sizes are $10^{\prime} \times 10^{\prime}$ and are extracted from the DSS-II-Red survey. In both cases, north is up and east to the left.
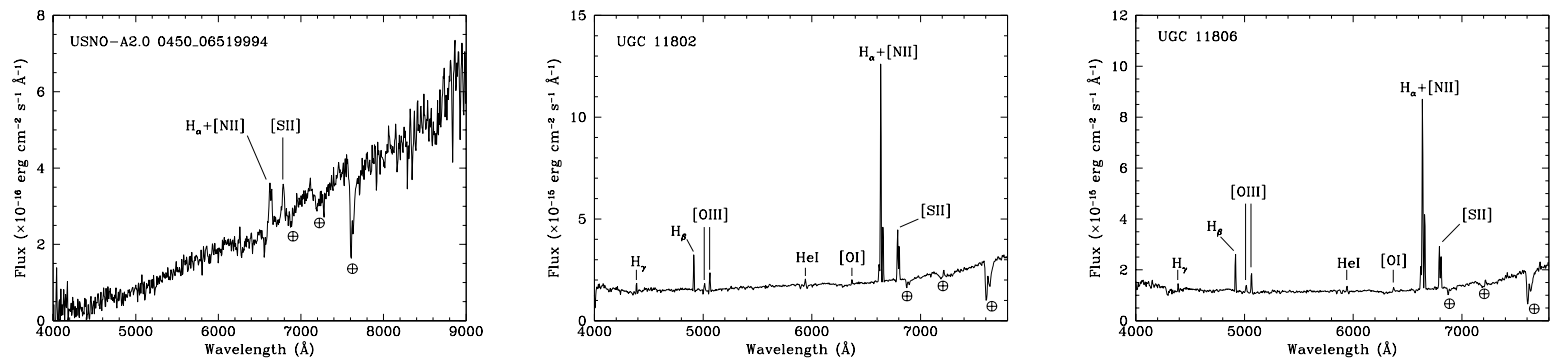

Figure 4: Spectra (not corrected for the intervening Galactic absorption) of the possible optical counterparts of the INTEGRAL sources IGR J08190-3835 (left panel) and IGR J21441+4640 (central and right panel). For each spectrum the main spectral features are labelled. The symbol $\oplus$ indicates atmospheric telluric absorption bands.

galaxy is the likely counterpart of the IBIS/BAT source and, on the basis of the detected X-ray absorption, we further suggest that it is a type 2 AGN.

\subsection{IGR J21441+4640}

Within the IBIS positional uncertainty of this source there are two galaxies (see Fig. 3, right panel), UGC 11802 and UGC 11806, at the same redshift ( $z=0.011$ ), which form the galaxy pair KPG559. Both galaxies are detected at radio frequencies in the NVSS catalogue and are also listed in the 2MASX Catalogue (see Fig. 2, bottom-right panel). The radio data analysis provides a 20$\mathrm{cm}$ flux for these objects of $\sim 23 \mathrm{mJy}$ and $11 \mathrm{mJy}$, respectively. Besides they are also detected at $92 \mathrm{~cm}$ and have spectral indices $\alpha=0.38$ and $\alpha=0.51$, respectively; both radio power and spectral index are typical of low luminosity AGN. Within the IBIS error box, XRT finds only one galaxy (UGC 11806) of the pair at $2.5 \sigma$ in the range $0.3-10 \mathrm{keV}$. The spectrum can be described by an unabsorbed power law with fixed photon index of 1.8 and an observed $2-10 \mathrm{keV}$ flux of $1.2 \times 10^{-13}$ $\mathrm{erg} \mathrm{cm}^{-2} \mathrm{~s}^{-1}$. The upper limit of the X-ray flux in the $2-10 \mathrm{keV}$ band for the companion galaxy UGC11802 is $8.3 \times 10^{-14} \mathrm{erg} \mathrm{cm}^{-2} \mathrm{~s}^{-1}$. Optical spectroscopy of UGC 11806 (Fig. 4, right panel) indicates that it is a narrow emission-line galaxy with flat continuum and prominent Balmer, $[\mathrm{N}$ 
II], [O III] and [S II] lines at a redshift consistent with the one found in the literature. Emissionline ratios suggest that this is a transition object, i.e. a LINER [10] with a possible contamination from an underlying starburst event. The optical spectrum of UGC 11802 (Fig. 4, central panel) indicates instead that this galaxy is a starburst, with no indication of AGN activity (which explains the absence of detectable X-ray emission from its nucleus). Overall we conclude that UGC 11806 is the possible counterpart of the IBIS source; it is probably a low-luminosity and highly variable AGN of the LINER type.

\section{Summary and Conclusions}

The basic idea of this work is to propose a way whereby AGN can be pinpointed among a set of unidentified objects detected in hard X-ray surveys. The method is tested here for a sample of eight objects, extracted from the fourth IBIS Survey Catalogue. We performed further studies including optical and/or X-ray follow-up observations, in order to verify their true association with the INTEGRAL source as well as their AGN nature and class. The optical spectra obtained for IGR J08190-3835 and IGR J21441+4640 allowed us to identify them as AGN belonging to the type 2 and LINER class. IGR J17520-6018 is most likely a type 2 AGN on the basis of the high X-ray absorption measured. Information regarding the other objects in the sample are fully discussed by Maiorano et al. (2011) and briefly summarized in the following. IGR J00556+7708, IGR J172191509 and IGR J21268+6203 3 are likely active galaxies on the basis of their radio spectra, NIR photometry and location above the Galactic plane. The radio/IR counterpart of IGR J05583-1257 is instead a starburst galaxy and so a less likely counterpart of the IBIS object, whereas in the case of IGR J03103+5706 we do not have sufficient information for a clear classification of the source.

\section{References}

[1] Bird A.J. et al., 2010, ApJS, 186, 1

[2] Skrutskie M.F. et al., 2006, AJ, 131, 1163

[3] Condon J.J. et al., 1998, AJ, 115, 1693

[4] Mauch T. et al., 2003, MNRAS, 342, 1117

[5] Stephen J.B. et al., 2005, A\&A, 432, L49

[6] Stephen J.B. et al., 2006, A\&A, 445, 869

[7] Stephen J.B. et al., 2010, MNRAS, 408, 422

[8] Maiorano E. et al., 2011, MNRAS, 416, 531

[9] Kalberla P.M.W. et al., 2005, A\&A, 440, 775

[10] Heckman T.M., 1980, A\&A, 87, 152 